\title{
The dual nature of the Milky Way stellar halo
}

\author{
Anna Curir ${ }^{1}$, Giuseppe Murante ${ }^{1}$, Eva Poglio ${ }^{2}$, Álvaro Villalobos ${ }^{3}$ \\ ${ }^{1}$ INAF- Astronomical Observatory of Torino, strada Osservatorio 20, 10025 Pino Torinese \\ (Torino) Italy \\ email: curir@oato.inaf.it \\ ${ }^{2}$ Dept. of Physics, Turin University, Italy \\ email: epoglio@studenti.ph.unito.it \\ ${ }^{3}$ INAF - Astronomical Observatory of Trieste,via Tiepolo 11, Trieste, Italy \\ email: villalobos@oats.inaf.it
}

\begin{abstract}
The theory of the Milky Way formation, in the framework of the $\Lambda$ CDM model, predicts galactic stellar halos to be built from multiple accretion events starting from the first structure to collapse in the Universe.

Evidences in the past few decades have indicated that the Galactic halo consists of two overlapping structural components, an inner and an outer halo. We provide a set of numerical N-body simulations aimed to study the formation of the outer Milky Way (MW) stellar halo through accretion events between a (bulgeless) MW-like system and a satellite galaxy. After these minor mergers take place, in several orbital configurations, we analyze the signal left by satellite stars in the rotation velocity distribution. The aim is to explore the orbital conditions of the mergers where a signal of retrograde rotation in the outer part of the halo can be obtained, in order to give a possible explanation of the observed rotational properties of the MW stellar halo.

Our results show that the dynamical friction has a fundamental role in assembling the final velocity distributions originated by different orbits and that retrograde satellites moving on low inclination orbits deposit more stars in the outer halo regions and therefore can produce the counter-rotating behavior observed in the outer MW halo.
\end{abstract}

Keywords. Galaxy: formation, kinematics and dynamics, halo

\section{Introduction}

Evidences in the past few decades indicated that the MW halo has a more complex structure than a single component. Recently Carollo et al (2007), analyzing a large sample of calibration stars from the Sloan Digital Sky Survey (SDSS), confirmed the existence of two broadly overlapping structural components -an inner and an outer halo- that exhibit different spatial density profiles, stellar orbits and stellar metalicities. In particular the inner halo shows a net prograde rotation around the center of the Galaxy. The outer halo, instead, shows a clear retrograde net rotation.

The theory of galaxy formation in a $\Lambda$ Cold Dark Matter $(\Lambda$ CDM) Universe predicts galactic stellar halos to be built from multiple accretion events starting from the first structures to collapse in the Universe. Halos, composed of both dark and baryonic matter, grow by merging with other halos. While the gas from mergers and accretions loses its energy through cooling and settles into a disk, the non dissipative material (accreted stars and dark matter (DM)) forms a halo around the Galaxy. We have many likely evidences of past accretion into the MW, therefore in this study we adopt the scenario in which the MW halo has been formed by subhalos accretion. We then focus on a possible origin 
of a retrograde outer stellar halo, defined as the set of stars orbiting around the Galaxy at large distance from the disk plane, namely $z>15 \mathrm{kpc}$. It is expected that such halo is made of stars stripped from merging or disrupted satellites of low mass.

With the aim to determine if, and in what condition, we can obtain a signal of retrograde rotation in the stellar distribution in the outer halo, we simulate minor mergers of a satellite halo with a main halo of Galactic mass (Murante et al. 2010). We used controlled and simplified numerical experiments instead of cosmological simulations, in order to focus on the dynamics of the outer halo, disentangling the effect of the pure gravitational dynamics from the complex gas physics that must be considered when performing self-consistent simulations of galaxy formation. Our simulations, are nevertheless cosmological motivated by the use of halos with characteristics in accord with cosmological simulations. After the satellite completes its merging/disruption process, we identify stars at distance larger than $15 \mathrm{kpc}$ from the disk plane and analyze their rotational velocities in order to determine if an excess of counter-rotating signal in the outer halo can be produced by couples of mergers having orbits with identical inclination and opposite initial rotation. We also investigate the possible influence of the spin parameter of the primary halo, the numerical resolution and the distribution of the stellar component, on the results.

\section{The Milky Way Stellar Halo}

A spiral galaxy like the Milky Way has three basic components in its visible matter: the bulge,the disk and the stellar halo. The galactic disk is indeed surrounded by a spheroid halo of old stars and globular clusters, of which $90 \%$ lie within $30 \mathrm{kpc}$, suggesting a stellar halo diameter of $60 \mathrm{kpc}$.

The observed rotation of the stars and gas clouds indicates that the visible matter is surrounded by a DM halo containing the major portion of the total galaxy mass and extending very far beyond the visible matter. Some indirect means suggest that the DM halo may extend as far as $170 \mathrm{kpc} / \mathrm{h}$ from the center of the galaxy.

According to Carollo et al. (2007), the inner-halo component of the MW dominates the population of halo stars found at distance up to $10-15 \mathrm{kpc}$ from the Galactic Center. An outer-halo component dominates in the region beyond 15-20 kpc. Inner-halo stars possess generally high orbital eccentricities, and exhibit a modest prograde rotation (between 0 and $50 \mathrm{~km} \mathrm{~s}^{-1}$ ) around the center of the Galaxy. The distribution of metallicities for stars in the inner halo peaks at $[\mathrm{Fe} / \mathrm{H}]=-1.6$. Outer-halo stars cover a wide range of orbital eccentricities, and exhibit a clear retrograde net rotation (between -40 and $-70 \mathrm{~km}$ $s^{-1}$ ) about the center of the Galaxy. The metallicity distribution function of the outer halo peaks at lower metallicity than that of the inner halo, around $[\mathrm{Fe} / \mathrm{H}]=-2.2$.

These properties indicate that the individual halo components probably formed in fundamentally different ways through successive dissipational (inner halo) and dissipationless (outer halo) mergers and tidal destruction of proto-Galactic clumps.

In our work we focus on the outer halo. To this purpose, we performed a set of numerical N-body simulations for studying the formation of the outer MW's halo through accretion events. After simulating minor mergers of a satellite with a DM main halo containing a stellar disk, we analyze the signal left by satellite stars in the rotational velocities distribution. Above all, we explore the orbital conditions starting from which a signal of retrograde rotation in the outer part of the halo can be obtained, in order to give a possible explanation of the rotational properties of the MW stellar halo. As we will discuss, the dynamical friction has a fundamental role in assembling the final velocity 
distributions originated by different orbits. Therefore, we will describe this effect in the next section.

\section{Dynamical Friction}

A particle of mass $M_{s}$ moving through a homogeneous background of individually much lighter particles with an isotropic velocity distribution suffers a drag force (Chandrasekar, 1943):

$$
F_{d}=-\frac{4 \pi G^{2} M_{s}^{2} \rho_{f}\left(<v_{s}\right) \ln \Lambda}{v_{s}^{2}}
$$

where $v_{s}$ is the speed of the satellite with respect to the mean velocity of the field and $\rho\left(<v_{s}\right)$ is the total density of the field particles with speeds less than $v_{s}, \Lambda$ is the Coulomb Logarithm (Binney and Tremaine, 1987).

It is expected that the outer halo is made of stars stripped from merging or disrupted satellites of low mass. It is very important to note that, from equation (3.1), we expect that, the higher is $v_{s}$, the weaker is the dynamical friction force. Retrograde satellites have higher $v_{s}$ with respect to prograde one, since in the first case velocity of the satellite is opposite to that of the disk and to rotational velocities of the main halo particles. As a consequence, prograde orbits decay faster. Particle stripped from the satellites will remain on the orbit on which the satellite was when they were stripped. How we can see from equation (3.1) the dynamical friction depends upon the satellite mass, so a single star does not feel the effect of this force, due to its to much small mass, and consequently conserves its energy remaining on the same orbit.

Another important gravitational effect, which happens during mergers, is the tidal disruption of satellites. While the tidal disruption is most important near the center of the main halo, where the gravitational potential is stronger, the dynamical friction is exerted both by the main halo DM particles and by the disk star particles.

\section{The simulations}

We perform two kinds of simulations. In both simulations, we use a primary DM halo with a NFW radial density profile (Navarro et al 1997), containing a stellar disk. What changes is the satellite: in the first set of simulations we realize a DM+bulge satellite configuration, in which the DM halo contains a stellar bulge, having an Hernquist (1990) radial density profile; in the second set the satellite is an halo with a NFW profile, in which we assume that the DM in the inner region traces the stars.

We simulated minor prograde mergers, in which a satellite co-rotates with the disk spin, and retrograde ones, with a counter-rotating satellite. Stars in the outer halo can be stripped from accreted DM satellites, hosting dwarf galaxies, during their orbits before the final merger. Minor mergers are the most probable sources for these kind of stars, firstly because they do not destroy the galactic disk and can thus happen in all evolutionary phases of the life of a disk galaxy. Moreover, a smaller satellite suffers less dynamical friction, and can deposit stars at larger distances from the galactic center, for a longer period of time.

We run all our simulation using the public parallel Treecode GADGET2 (Springel, 2005).

Into our halo, we embed a truncated stellar disk supported by rotation, having an exponential surface density law. The disk is in gravitational equilibrium with the DM halo. 
An analytical mass model for spherical galaxies and bulge has been proposed by Hernquist (1990). This model is one of the most successful analytic model for these stellar systems. It as a $\rho \propto r^{-4}$ profile in the outer parts and a central density cusp of strength $\rho \propto r^{-1}$.

In the first set of simulations, in which we used a DM+bulge satellite configuration, we performed a suite of numerical experiments only in high resolution. In the second one, in which the satellite is a DM halo with a NFW profile, we run a suite of numerical experiments, varying the force and mass resolution of both the primary and the secondary.

We choose our minor mergers with a mass ratio $M_{\text {prim }} / M_{\text {sat }} \approx 40$, similar to the estimated mass ratio of the Large Magellanic Cloud (LMC) to the MW halo.

All the physical parameter of the main DM halo, of the DM satellite, of the DM+bulge satellite and of the main disk are listed in Tables 1, 2, 3 and 4.

\begin{tabular}{cccccccc}
\hline Halo & $M_{200}$ & $R_{200}$ & $C_{200}$ & $R_{\text {trunc }}$ & $\mathrm{N}$ & $\epsilon$ & $M_{D M}$ \\
\hline Main-HR & $10^{12}$ & 165 & 7.25 & 1300 & $10^{6}$ & 0.5 & $10^{6}$ \\
\hline Main-LR & $10^{12}$ & 165 & 7.25 & 1300 & $10^{5}$ & 1.0 & $10^{7}$ \\
\hline Satellite-HR & $2.4 \cdot 10^{10}$ & 47 & 8.54 & 400 & $10^{5}$ & 0.5 & $2.4 \cdot 10^{5}$ \\
\hline Satellite-LR & $2.4 \cdot 10^{10}$ & 47 & 8.54 & 400 & $10^{4}$ & 1.0 & $2.4 \cdot 10^{6}$ \\
\hline
\end{tabular}

Table 1. Properties of main and satellite halos (NFW profile).Column 1: Halo. Column 2: Virial mass in $\left(M_{\odot} / h\right)$; here we refer all our virial quantities to an overdensity of 200 times the mean cosmic density. Column 3: Virial radius in $(k p c / h)$. Column 4: NFW concentration parameter $\left(c_{200}=r_{200} / r_{s}\right)$. Column 5: Truncation radius. Column 6: Softening length in $(k p c / h)$. Column 7: Mass of DM particle in $\left(M_{\odot} / h\right)$.

\begin{tabular}{ccccccc}
\hline Halo & $M_{200}$ & $R_{200}$ & $C_{200}$ & $\epsilon$ & $a$ & $M_{b}$ \\
\hline Satellite-stars & $2.4 \cdot 10^{10}$ & 47 & 8.54 & 0.25 & 0.709 & $2.4 \cdot 10^{9}$ \\
\hline
\end{tabular}

Table 2. Properties of the satellite halo (Hernquist profile). Column 1: Halo. Column 2: Virial mass in $\left(M_{\odot} / h\right)$. Column 3: Virial radius in $(k p c / h)$. Column 4: NFW concentration parameter. Column 5: Softening length in $k p c / h$. Column 6: Hernquist scale radius in $k p c / h$. Column 7 : Mass of the stellar bulge in $M_{\odot} / h$. In the case of the Hernquist profile, the secondary halo has an exponential cut-off in density.

\begin{tabular}{ccccc}
\hline Halo & $N_{D M}$ & $N_{*}$ & $M_{D M}$ & $M_{*}$ \\
\hline Satellite-stars & $1.1 \cdot 10^{5}$ & $10^{5}$ & $1.95 \cdot 10^{5}$ & $2.38 \cdot 10^{4}$ \\
\hline
\end{tabular}

Table 3. Properties of the satellite halo (Hernquist profile). Column 1: Halo. Column 2: DM particles number inside the virial radius. Column 3: Star particles number inside the virial radius. Column 4: Mass of DM particle in $\left(M_{\odot} / h\right)$. Column 5: Mass of star particle in $\left(M_{\odot} / h\right)$.

We put our satellite on a prograde orbit, in which the satellite co-rotates with respect to the disk spin, and a retrograde (counter-rotating) one. We choose two orbits used in Read et al. (2008) for studying the thickening of the disk due to the same kind of minor merger: a low-inclination one, with a 10 degree angle with the disk plane, and a high inclination one with a 60 degree angle.

Initially, the center of the primary halo stays in the origin of our coordinate system. The satellite coordinates and its components of the velocity are listed in Table 5. 


\begin{tabular}{ccccccc}
\hline Disk & $M_{*}$ & $r_{\text {disk }}$ & $r_{0}$ & $\mathrm{~N}$ & $\epsilon$ & $Q$ \\
\hline Disk-HR & $5.7 \cdot 10^{10}$ & 20 & 4 & $10^{6}$ & 0.5 & 2 \\
\hline Disk-LR & $5.7 \cdot 10^{10}$ & 20 & 4 & $10^{5}$ & 1.0 & 2 \\
\hline
\end{tabular}

Table 4. Properties of the disk. Column 1: Disk. Column 2: Disk stellar mass in $\left(M_{\odot} / h\right)$. Column 3: Disk truncation radius in $(k p c / h)$. Column 4: Disk scale radius in $(k p c / h)$. Column 5: Particles number inside the disk radius. Column 6: Softening length in $(k p c / h)$. Column 7: Toomre parameter.

\begin{tabular}{cccccccc}
\hline & Orbit & $x$ & $y$ & $z$ & $v_{x}$ & $v_{y}$ & $v_{z}$ \\
\hline Pro1 & Prograde-1 $\left(10^{\circ}\right)$ & 80.0 & 0.27 & -15.2 & -6.3 & 62.5 & 0.35 \\
\hline Ret1 & Retrograde-1 $\left(10^{\circ}\right)$ & 80.0 & 0.27 & -15.2 & -6.3 & -62.5 & 0.35 \\
\hline Pro2 & Prograde-2 $\left(10^{\circ}\right)$ & 29.5 & 0.27 & -5.2 & -6.3 & 89.3 & 0.35 \\
\hline Ret2 & Retrograde-2 $\left(10^{\circ}\right)$ & 29.5 & 0.27 & -5.2 & -6.3 & -89.3 & 0.35 \\
\hline Pro & Prograde $\left(60^{\circ}\right)$ & 15.0 & 0.12 & -26.0 & -1.2 & 80.1 & 2.0 \\
\hline Ret & Retrograde $\left(60^{\circ}\right)$ & 15.0 & 0.12 & -26.0 & -1.2 & -80.1 & 2.0 \\
\hline
\end{tabular}

Table 5. In the first three columns, are listed the initial satellite coordinates in $k p c$, while in the last three the components of velocity in $\mathrm{km} / \mathrm{s}$. At low inclination, we simulate mergers with two different departures, one further from the disk center (1) and one nearer to the disk center (2).

In our full suite of numerical experiments, we vary the force resolution and the DM angular momentum of both the primary and the secondary. In our first set of simulation, our secondary halo always has a spin parameter $\lambda=0$, where we define

$$
\lambda=\frac{J}{\sqrt{2} M V R}
$$

whit $M$ being the mass inside a radius $R$ and $V=G M / R$ the circular velocity, as in Bullock et al.(2001).

We use simulations with a primary halo with a spin parameter $\lambda=1$ (A case) and $\lambda=0$ (B case). We assign the angular momentum to DM particles using a rigid body rotation profile. In our main halo, the angular momentum of DM particles is always aligned with that of stellar disk.

In our second set of simulations, we repeated our experiments using a Bullock's profile instead of a rigid rotation one for the DM rotation velocity (only in the case in which the secondary halo has a DM+bulge configuration). Bullock et al.(2001) found indeed that in cosmological simulations the specific angular momentum $j$ follows a power law: $j(r) \propto r^{\alpha}$ with $\alpha$ roughly distributed over the halos like a Gaussian. In this case, we set the spin parameter of the DM halo to $\lambda=0.06$, a value suggested by cosmological simulations.

We note that the average rotational speed of haloes having a Bullock profile with $\lambda=0.06$ and a rigid rotation profile with $\lambda=1$ are of the same order of magnitude in the inner part, where the disk resides.

In the DM-only simulations the satellite is a DM halo with a NFW radial density profile. In this set of simulations, we follow the evolution of the inner region of the satellite, in particular a sphere of $8 \mathrm{kpc}$ radius, centered on the center of mass of the satellite, and we assume that the DM particles in this inner regions trace the stars. The 
choice of such a value is due to the fact that, the gravitational mass contained within $8 \mathrm{kpc}$ radius, is comparable with the gravitational mass of the LMC. Besides, since the NFW profile tends to $r^{-3}$ at $r>r_{s}$, in good approximation one can consider that the majority halo mass is contained in the inner regions. We run our DM-only numerical experiments, varying the mass and force resolution in both the primary and the secondary: the case of LR has $10^{4}$ particles within the virial radius of the DM satellite, whereas in the case of $\mathrm{HR}$ we have $10^{5}$ particles within the virial radius of the DM satellite.

The time unit of the code is $T_{*}=0.98$ Gyrs. We run all our simulations for $T=$ $4.725 T_{*}$, corresponding to $\sim 16$ dynamical time of the main halo. We define a merger to be completed when the $z$ coordinate of the center of mass of all satellite stars remains within $2 \mathrm{kpc}$ from the initial disk plane.

The simulations were carried out at CASPUR, with CPU time assigned with the "Standard HPC grant 2009", and at the CINECA, Bologna, with CPU time assigned under an INAF/CINECA grants.

\section{Distribution of $v_{\Phi}$}

In order to evaluate the $v_{\Phi}$ we rotate the coordinate system so that the disk lies in the XY plane. In Figure 1, we show histograms of the rotation velocity obtained in the

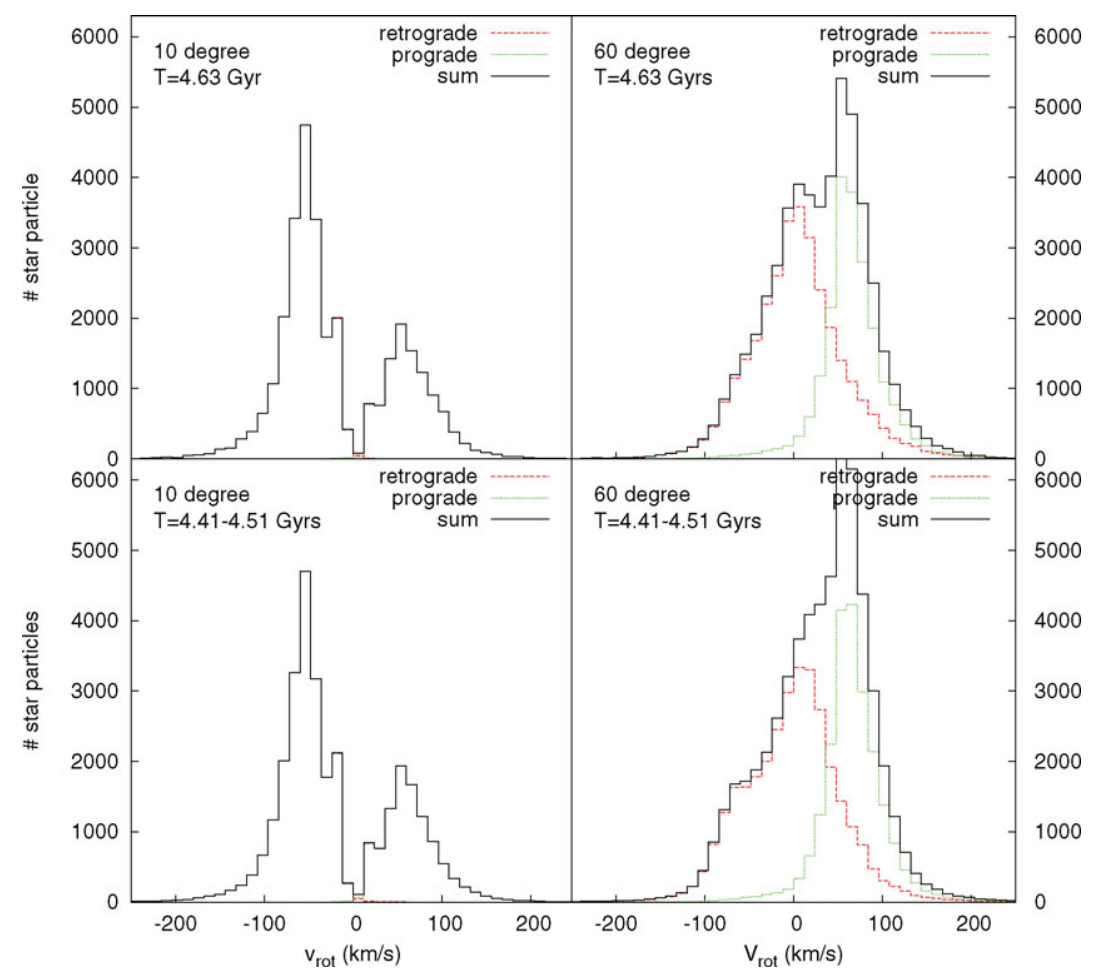

Figure 1. Histograms of rotation velocities for star particles in the outer halo, at the end of the set A of merger simulations. Upper panels show the histograms at the final time, lower panel show the same histograms, averaged over five simulation snapshots. In red (dashed line), we plot the histogram obtained for our retrograde orbits; in green (dotted line), those for our prograde orbits. In black (solid line) we show the sum of the two. Left column is for the low inclination orbits, right column for the high inclination ones. We used 50 velocity class, equispaced, between -300 and $300 \mathrm{~km} / \mathrm{s}$ in all histograms. 
four simulations of the set A: the satellite is either co-rotating or counter-rotating with respect to the disk, and the orbit has either a low or a high inclination with respect to the disk plane. In the upper panels, we show histograms at the final time of our simulations; in the lower ones, we performed an average over five consecutive snapshots, which cover a period of time of $100 \mathrm{Myr}$, to get rid of possible sampling effect on the particle orbits which can rise using a particular instant of time. We show both the distributions of stars from single simulations, and the one resulting from taking together star particles from prograde and retrograde orbits having the same initial inclination. The latter gives us a hint on the possible origin of the rotation signal in the case a similar number of prograde and retrograde minor accretion events happen during the formation history of a galaxy, under the hypothesis that their inclination is the same. It is immediately clear from the Figure1 that our pair of low inclination orbits show an excess of counter-rotating stars in the outer halo. It remains to be determined if the counter-rotating signal is caused by the interaction with the stellar disk of the main halo, or with the DM of the halo itself.

Figure 2 shows the results of the same analysis performed on our set A, but in the case in which no halo spin is present (set B). Here we only show the results for our last snapshot; as in Figure 1, averaging over five snapshots makes no appreciable difference. Also in our set B, low inclination retrograde orbits produce more counter-rotating star

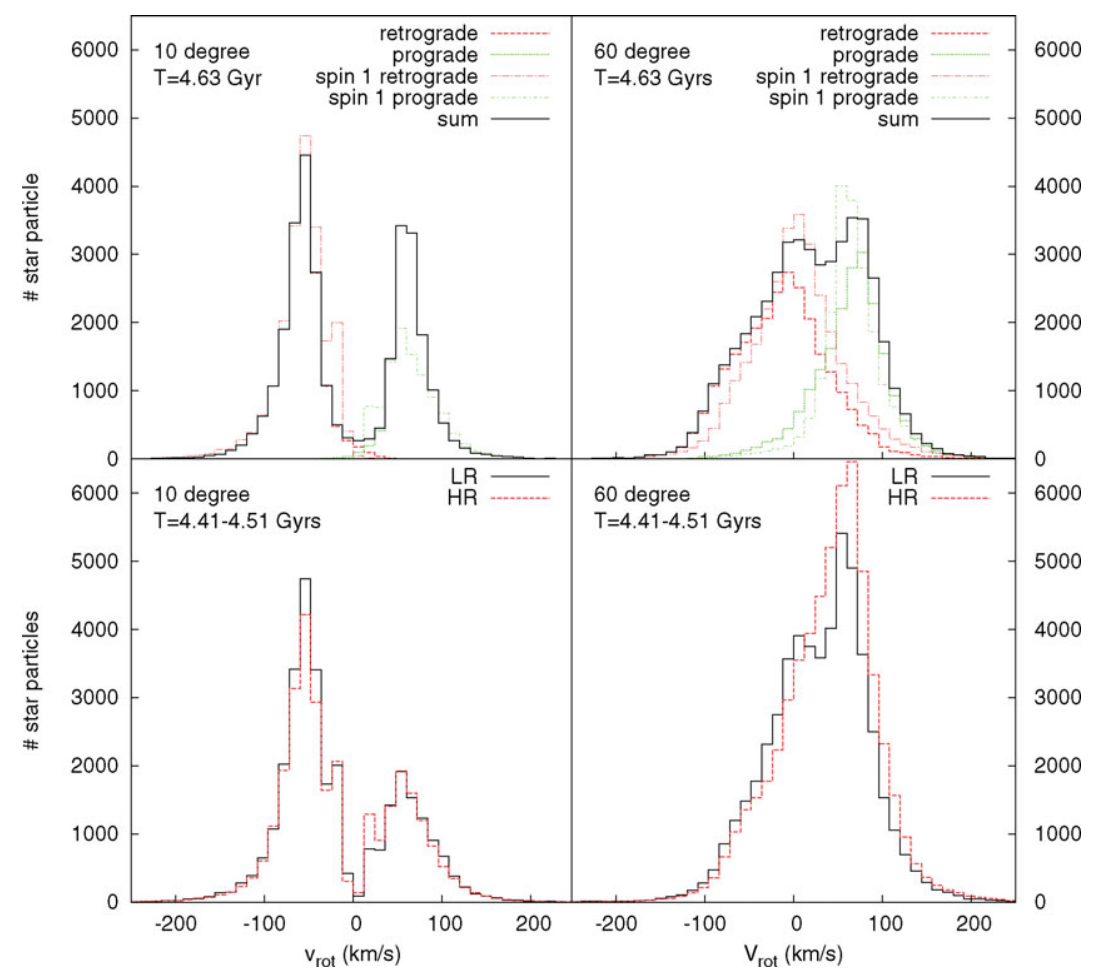

Figure 2. Upper row: histograms of rotation velocities for star particles in the outer halo, at the end of the set B of merger simulations, in which the main halo has no spin. In red (dashed line), we plot the histogram obtained for our retrograde orbits; in green (dotted line), those for our prograde orbits. In black (solid line) we show the sum of the two. Left panel is for the low inclination orbits, right panel for the high inclination ones. We also show the histogram for the case of spin 1 (dashed-dotted lines). Lower row: histogram of rotation velocities for star particles in the outer halo, for our set A, at the basic resolution ("LR", black continuous line) and at a ten times better mass resolution ("HR", red dashed line). 
particle than co-rotating stars produced by prograde orbits. Again, this is not the case for high inclination orbits. From Figure 2, it is clear that the excess of counter-rotating star is anyway smaller: and this is due to the fact that more co-rotating stars are produced by prograde orbit in our no spin case than in spin 1 case.

Therefore, both disk rotation and halo spin contribute to the slowing-down of prograde orbits and to the consequent smaller amount of high-energy star particle stripped from satellites that can reach the outer halo. However, disk rotations appear to be the main driver of such an effect.

As far as the resolution comparison is concerned, in the lower row of Figure 2 we note that our result does not depend on the mass resolution of our satellite halo. Even using 10 times more particles in the secondary, only our low inclination couple of mergers shows an excess of counter-rotating stars in the outer halo.

\section{Conclusions}

Our main results are the following:

- low inclination mergers do produce an excess of counter-rotating satellite stellar particles in the outer halo, independently on the spin of the DM;

- high inclination orbits do not seem to produce a significant counter-rotating signal;

- the fraction of counter-rotating to co-rotating satellite stars in the outer stellar halo is higher if the DM has spin.

In the $\Lambda \mathrm{CDM}$ model, there is no reason for expecting an excess of retrograde mergers (Sales et al. 2008). Thus, if such an excess was needed to produce a counter-rotating signal in the outer stellar halo, we should require a peculiar accretion history for the MW. The main result of this work shows that such a fine-tuning in the MW history is not needed. Even if, statistically, the number of prograde and retrograde minor merger is the same, still a counter-rotating signal can arise if such mergers predominantly happen along low inclination orbits. Since matter accretion, in a CDM dominated Universe, mainly occurs along filaments, this will be the case if the galaxy disk is co-planar to the (majority of) filaments. The disk-filament alignment issue is still debated (Brunino et al. 2007): from our results, we expect that if the galactic disk were perpendicular to the main accretion streams, no counter-rotating signal should be observed in the outer halo star distribution.

\section{References}

Carollo, D., Beers, T., C., Sun Lee, Y., Chiba, M., E. Norris, J. E., Wilhelm, R., Sivarani, T., Marsteller, B., Munn, J., A., Bailer-Jones, C., A., L., Re Fiorentin, P. \& York, D., G. 2007, Nature, 450, 1020

Murante, G., Poglio, E., Curir, A. \& Villalobos, A. 2010, ApJ (Letters), 716, L115

Chandrasekar, S. 1943, ApJ, 97, 255

Binney and Tremaine 1987, in: Galactic Dynamics (Princeton, NJ: Princeton University Press), p. 427

Navarro, J. F., Frenk, C. S. \& White, S. D. M. 1997, ApJ, 190, 493

Hernquist, L. 1990, ApJ, 356, 359

Springel, V. 2005, ApJ, 364, 1105

Read, J. I., Lake, G., Agertz, O. \& Debattista, V. P. 2008, MNRAS 389, 1041

Bullock, J., Dekel, A., Kolatt, T., Kravtsov, A.,V., Klypin, A. \& Porciani, C. and Primack, J. 2001, ApJ, 555, 240

Sales, L., V., Helmi, A., Starkenburg, E., Morrison, H., L., Engle, E., Harding, P., Mateo, M., Olszewski, E., W. \& Sivarani, T. 2008, MNRAS 389, 1391

Brunino, R., Trujillo, I., Pearce, F. R. \& Thomas, P. A. 2007, MNRAS 375, 184 